\title{
Time-resolved velocity and pressure field quantification in a flow-focusing device for ultrafast microbubble production
}

\author{
Sarah Cleve $\odot,{ }^{1}$ Christian Diddens $\odot,{ }^{1}$ Tim Segers $\odot,{ }^{2}$ \\ Guillaume Lajoinie $\odot,^{1}$ and Michel Versluis $\oplus^{1}$ \\ ${ }^{1}$ Physics of Fluids Group, MESA+ Institute for Nanotechnology and Technical Medical (TechMed) Center, \\ University of Twente, P.O. Box 217, 7500 AE, Enschede, The Netherlands \\ ${ }^{2}$ BIOS Lab-on-a-Chip Group, Max-Planck Center Twente for Complex Fluid Dynamics, MESA+Institute for \\ Nanotechnology, University of Twente, P.O. Box 217, 7500 AE, Enschede, The Netherlands
}

(Received 30 June 2021; accepted 26 October 2021; published 15 November 2021)

\begin{abstract}
Flow-focusing devices have gained great interest in the past decade, due to their capability to produce monodisperse microbubbles for diagnostic and therapeutic medical ultrasound applications. However, up-scaling production to industrial scale requires a paradigm shift from single chip operation to highly parallelized systems. Parallelization gives rise to fluidic interactions between nozzles that, in turn, may lead to a decreased monodispersity. Here we study the velocity and pressure field fluctuations in a single flow-focusing nozzle during bubble production. We experimentally quantify the velocity field inside the nozzle at $100 \mathrm{~ns}$ time resolution, and a numerical model provides insight into both the oscillatory velocity and pressure fields. Our results demonstrate that, at the length scale of the flow-focusing channel, the velocity oscillations propagate at fluid dynamical timescale (order of $\mu \mathrm{s}$ ) whereas the dominant pressure oscillations are linked to the bubble pinch-off and propagate at a much faster timescale (order of ns).
\end{abstract}

DOI: 10.1103/PhysRevFluids.6.114202

\section{INTRODUCTION}

Coated microbubbles are currently investigated for a broad variety of medical applications, both in the fields of diagnostic imaging and therapy [1], which include sonoporation [2], blood-brain barrier opening [3], and sonothrombolysis [4]. The diagnostic application relies on the scattered nonlinear echo and therapy requires a mechanical response of the microbubbles to the driving ultrasound. Both the echo and volumetric oscillation amplitude depend on the driving frequency with respect to the resonance frequency of the bubble [5], which is inversely proportional to the bubble size. Clinically available ultrasound contrast agents contain microbubbles with a broad size distribution, typically ranging from 1 to $10 \mu \mathrm{m}$ in diameter [6]. A narrowband ultrasound wave typically used for therapy and diagnostics would therefore effectively drive only a small fraction of the contrast agent population contained in the injected dose. This suboptimal match between ultrasound spectral content and bubble size distribution motivates the development and use of monodisperse microbubbles. The increased sensitivity of monodisperse bubbles with respect to polydisperse ones has first been demonstrated for molecular imaging using low bubble concentrations [7] and later for contrast imaging in vitro [8] and in vivo [9]. In particular, Segers et al. [8] report sensitivity increase by two and three orders of magnitude for the fundamental and second harmonic, respectively.

In order to obtain a monodisperse contrast agent, one can either extract bubbles of the desired size from a polydisperse suspension, e.g., by acoustic sorting [10] and differential centrifugation [11], or one can create monodisperse bubbles directly, e.g. in a microfluidic device. In the past two decades, different microfluidic techniques have been investigated for the production of monodisperse bubbles 
including cross-flow, co-flow, and flow-focusing as summarized in [12]. The use of flow focusing is particularly promising as it allows for production rates exceeding 1 million bubbles per second $[13,14]$. Flow-focusing devices can be operated in three different regimes [15]: (1) In the squeezing regime, also called geometrically controlled break up, bubbles completely block the outlet channel or orifice before pinch-off. (2) In the dripping regime a gas jet is formed, but its tip retracts periodically from the outlet channel. (3) In the jetting regime, the gas jet stably extends into the flow-focusing channel, and bubbles pinch off from its tip. Among these regimes, the jetting regime is characterized by the highest production rates. However, even at a production rate of 1 million bubbles per second a single nozzle can produce only a few clinical doses per hour. In order to reach industrially relevant quantities, upscaling is necessary.

Upscaling by replicating the driving and control systems necessary to drive a single chip is prohibitive in terms of cost, operation difficulty, and space. An evident approach to upscaling is therefore the parallelization of multiple nozzles. Such a strategy has been investigated for parallel droplet production $[16,17]$, which has then been translated to upscaled bubble production with some success, using two to several hundred nozzles [18-23]. Despite the impressive achievements on the production rate $[22,23]$ the optimal parameter range for stable bubble production can hitherto only be extracted from empirical methods. Furthermore, the different parallelization strategies explicitly $[19,21]$ or implicitly show a loss of monodispersity as compared to a single nozzle system. In comparison, monodispersity seems to be less affected in parallel droplet production [24]. Possible reasons are the compressibility of the gas phase but also the fact that the liquid flow is typically mass flow controlled while the gas flow is mostly pressure controlled. In studies where the supply lines were connected [25], only weak interactions were reported for droplet production.

The increased polydispersity during bubble production is suspected to arise from the communication between nozzles [24] through the common liquid and gas supply lines, as well as through as the common outlet channel. Two possible sources of communication between channels have been discussed in literature [22,24]. The first one consists in purely geometrical considerations such as varying hydraulic resistances of individual channels due to, e.g., design or fabrication inaccuracies. These geometrical differences can be alleviated by a thorough design of a completely symmetric system $[20,21]$ or by adapting the size of each channel to ensure equal flow resistances for each nozzle [22]. The second type of communication, the so-called "cross-talk," refers to oscillatory flow phenomena in one nozzle propagating to the neighboring nozzles and is more difficult to eliminate or control, a priori. Evidence for cross-talk during bubble production in the squeezing regime has been reported by Hashimoto et al. [18,24]. Their system has a common outlet channel and separate, decoupled inlets for two to four nozzles. There, bubbles in neighboring nozzles are produced in antiphase. The authors argue that a change in flow resistance during bubble production directly influences the flow rate provided to the other channel(s), which then leads to an alternating bubbling regime.

Crosstalk between parallelized channels due to flow and pressure fluctuations at the timescale of microseconds seems a likely candidate for influencing the monodispersity of the produced bubbles. However, studies have so far been conducted only for the squeezing regime. The role of time-resolved cross-talk between parallelized channels in the jetting regime, has not yet been studied, to the best of our knowledge. However, some existing studies have considered the effect that externally imposed oscillations have on the production of bubbles. The influence of an acoustic pressure wave inside the gas supply line has been studied by Shirota et al. [26,27] for the pinch-off of submillimetric bubbles into a quiescent liquid. Shirota et al. [26] argue that a temporarily increased gas inflow caused by the short ultrasound pulse will promote the bubble pinch-off, while Shirota et al. [27] make the negative pressure pulse responsible for this. Even though the time and length scales in our flow-focusing device differ by several orders of magnitude compared to the system used by Shirota et al. [26,27], it is likely that pressure pulses do have an influence on bubble production. To the best of our knowledge, no studies have investigated the effect of forced oscillations of the liquid flow on the timescale of the production frequency of bubbles. Such studies have however been conducted for droplets, numerically by $\mathrm{Mu}$ et al. [28] and experimentally by Yang et al. [29]. The 


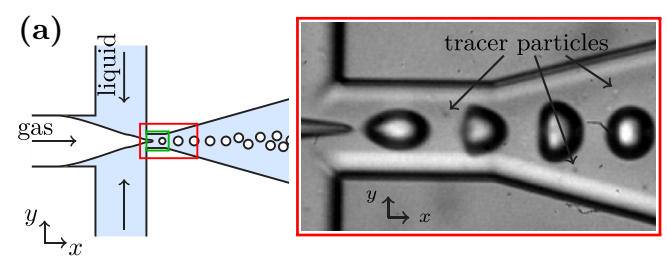

(b)
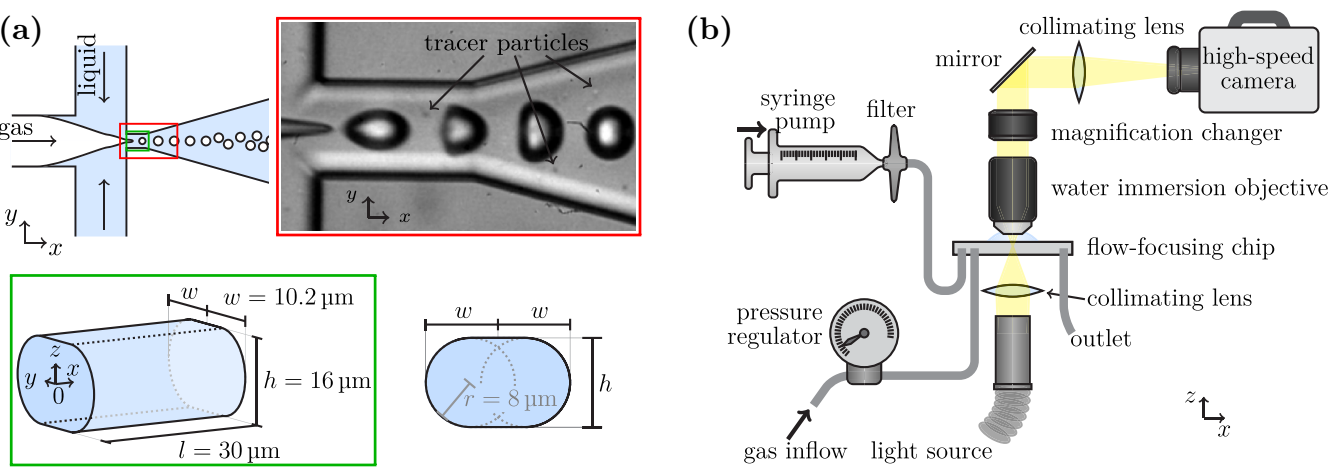

FIG. 1. (a) Schematic of the flow-focusing region with typical snapshot from a high speed recording and explanation of the geometry of the flow-focusing channel and its cross section. (b) Schematic of the experimental setup.

authors find that the droplet production frequency will lock into the actuation frequency as long as the two are not too different; in that case the production becomes polydisperse but periodic (binodal, Hopf bifurcation) or even completely unpredictable, probably chaotic. Furthermore, actuating both inner and outer liquid during droplet production has an effect on droplet size [28,29]. If velocity fluctuations are strong enough to play a role in the coupling between several flow-focusing nozzles for bubble production, one could expect a favorable locking to one and the same frequency, although the exact phase behavior may be both, either beneficial of unfavorable. Another type of study [30,31] has shown that oscillations would let particles migrate towards equilibrium positions inside the channel, however typically requiring timescales much larger than available in the present system.

Before analyzing typically complex parallelized systems, we propose to understand the nature and magnitude of the oscillatory components in a simpler device with a single flow-focusing nozzle. The unsteady nature of the bubbles is easily observed from time-dependent gas-liquid interface, but fluctuations also propagate through the gas and liquid phases. Understanding the unsteady components may aid the development of more complex systems with parallel flow-focusing nozzles. The present study characterizes the pressure and velocity field in a single flow-focusing nozzle. Ultra-high-speed imaging measurements combined with particle tracking velocimetry give access to the velocity field; the experimental methods are introduced in Sec. II. Numerical simulations, Sec. III, confirm these findings and further allow the detailed study of the dynamic pressure field. For both velocity and pressure, the field can be separated into (1) a time-averaged field, Sec. IV A, which can be compared to simplified theoretical models, and (2) oscillatory components, Sec. IV B, which reflect the unsteady nature of the bubble production. We show that the liquid velocity oscillations are induced by an oscillating gas flow rate and the periodic bubble pinch-off, and that they propagate at fluid dynamics timescale of microseconds. Pressure oscillations on the other hand, are dominated by a strong pressure pulse linked to bubble pinch-off and propagate through the flow-focusing region at the timescale of nanoseconds. A discussion, Sec. V, and conclusion, Sec. VI, conclude this paper.

\section{EXPERIMENTS}

\section{A. Experimental setup}

Experiments were conducted on a flow-focusing device that was fabricated by isotropic etching of the channel features in two glass wafers (i.e., with hydrofluoric acid etching at the same rate in all directions and thus creating corners with a radius equal to the etching depth [32]). These were then aligned and bonded together as described in Segers et al. [33]. The channel section where 
the bubbles are being formed [see Fig. 1(a)] has a width $b=2 w=20.4 \mu \mathrm{m}$ and height $h=2 r=$ $16.0 \mu \mathrm{m}$. The length of this section is $l=30.0 \mu \mathrm{m}$.

The liquid used in the present experiments was water with a weight fraction of $2 \%$ Tween 80 (Sigma-Aldrich). The surfactant was added to decrease the probability of bubble coalescence downstream in the expanding outlet [34]. This allowed a larger parameter range for stable bubble production. In the nozzle region, Tween 80 has a similar stabilizing effect on the bubble production as phospholipids that are typically used to stabilize medical ultrasound contrast microbubbles. Tracer particles (red fluorescent microspheres, $0.52 \mu \mathrm{m}$ in diameter, Thermo Scientific) were added with a weight fraction of approximately $1 \%$ to visualize the flow field. The liquid was supplied to the chip with a syringe pump (Harvard) operated at a constant flow rate. A filter (Acrodisc PSF Versapor $10 \mu \mathrm{m}$, Pall) mounted between the pump and the chip was used to prevent clogging by dust particles. The employed gas was air, provided by a laboratory internal pressure supply. A pressure regulator (IMI Norgren) was used to control the input gas pressure. The chip was connected to the inlets via a chip holder (Fluid Connect PRO, Micronit), and the nozzle region was visualized using a bright-field modular microscope (Olympus, BXF) equipped with a $60 \times$ water-immersion objective (LUMPlanFl, $0.90 \mathrm{NA}$, Olympus), an additional $2 \times$ magnification unit (U-ECA, Olympus), and a mirror (U-DP, Olympus). The total imaging magnification was $120 \times$. A small water drop was placed on top of the chip for optical coupling between the chip and the water-immersion objective. High-speed recordings were acquired with an ultra-high-speed camera (Shimadzu HPV-X2) operated at a frame rate of 10 million frames per second and a resolution of $400 \times 250$ pixels. The imaging resolution was $0.25 \mu \mathrm{m} /$ pixel, which was calibrated with a USAF 1951 calibration grid. A Xenon strobe light (Vision Light Tech), synchronized with the camera and coupled through an optical fiber and condensing lens provided sufficient light intensity for the optical recording. Each recording allowed us to save 256 images. However, owing to the short duration of the light pulse of $\sim 15 \mu \mathrm{s}$, only about half of these recorded images were bright enough for further processing.

\section{B. Experimental procedure}

The chip can be operated at different pressures and liquid flow rates. In the present paper, we focus on flow rates ranging from $150 \mu \mathrm{L} \mathrm{min}^{-1}$ to $250 \mu \mathrm{L} \mathrm{min}^{-1}$ and pressures ranging from $1.8 \mathrm{bar}$ to 3.2 bar. For each measurement series, the parameters were fixed and the chip was operated for several minutes in order to ensure a stable bubble production. In order to have sufficient sample data to evaluate the mean velocity field via particle tracking, each measurement series consisted of at least 100 recordings. Subsequently, 400 recordings for each parameter set were saved to extract the time-resolved velocity field. Due to the data transfer time of the camera, the total duration of a single measurement series ranged from 15 to $80 \mathrm{~min}$.

\section{Bubble detection}

The particles and noise were filtered out by means of singular value decomposition (SVD) by keeping only the large coherent structures of the image series. The bubble shapes were then extracted by applying a threshold. All extracted information (bubble size, shape, and position) were referenced to the previous pinch-off event so that all temporal information is contained within the time span $\left[t=0, t=1 / f_{b}=T_{b}\right]$, where $f_{b}$ and $T_{b}$ are the bubble production rate and period, respectively. The bubble production rates obtained for the present data range from $0.65 \times 10^{6}$ to $1.3 \times 10^{6}$ bubbles per second, with radii ranging from $3.5 \mu \mathrm{m}$ to $6.0 \mu \mathrm{m}$. The bubble volume was obtained through volume integration of the bubble contour assuming axial symmetry along the $x$-axis.

\section{Particle tracking and flow velocimetry}

SVD filtering keeping only small coherent structures and subsequent noise filtering and thresholding were applied to extract the positions of the particles. The positions were then developed into 


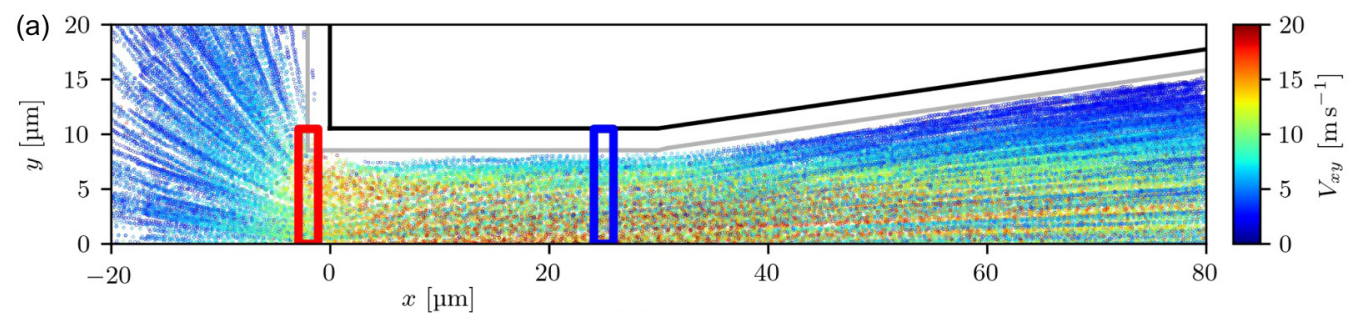

(b)

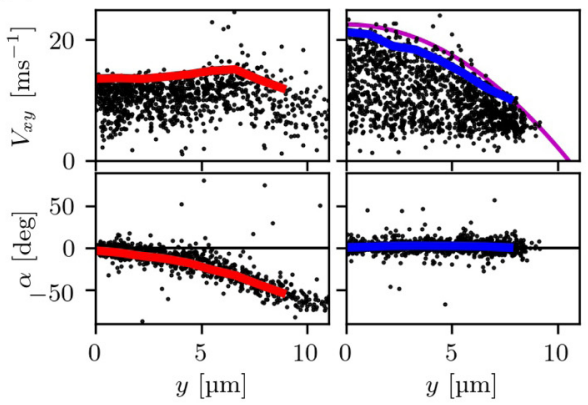

(c)

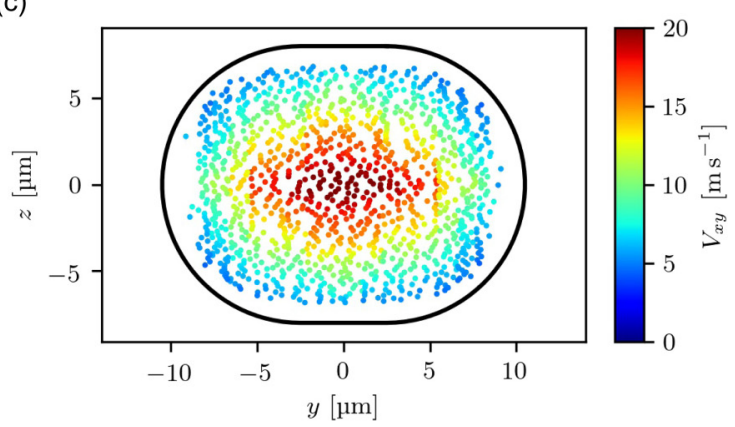

FIG. 2. Visualization of the postprocessing. (a) More than 130000 data points for the tracer particles presented along the channel profile. The total particle velocity $V_{x y}$ is indicated by the color code. The gray line indicates the border of the shadow region of the channel wall, where no particles can be detected. (b) Total velocity $V_{x y}$ and flow direction $\alpha$ for the two sections marked by the rectangles in (a). The colored bold lines correspond to the velocities and angles obtained through postprocessing for the $2 \mathrm{D}$ imaging plane. The thin, magenta line indicates a Poiseuille flow for comparison. (c) Reconstruction of the velocity in the cross section $x \approx 25 \mu \mathrm{m}$, based on the assumption that the velocity is maximum in the channel center and that detected tracer particles are equally spaced in the $z$-direction for a given position $y$.

trajectories with the help of the Matlab script track [35]. The velocities were subsequently calculated from the temporal difference of the particle positions between successive snapshots. As for the bubbles, all information was related to the bubbling period $T_{b}$. An example of all extracted sets of information for a flow without bubbles, totaling over 130000 data points, is shown in Fig. 2(a). The large variation in tracer velocities at a single location $(x, y)$ reflects the 3D structure of the flow that has a low velocity near the top and bottom walls $(|z| \approx r)$ and a maximum velocity in the channel center $(z \approx 0)$. The theoretical depth of field (DOF) of the $60 \times$ objective is limited to $\pm 1.6 \mu \mathrm{m}$. Nonetheless, out-of-focus tracer particles located at much larger distances from the focal plane are also captured as blurred entities. The recordings thus gather information on the $3 \mathrm{D}$ velocity field. While the experimental data do not allow a direct evaluation of the $z$-position, we can reconstruct the data as a function of $z$ by assuming that (1) velocity is maximum in the center of the channel and (2) that the particles are equally distributed in the flow; see Fig. 2(c). The main interest for the present study lies in extracting the velocity field in the central imaging plane $(z=0)$, where bubbles are passing. An example is shown in Fig. 2(b) for two-channel cross sections. The maximum velocity, assumed to lie in the central plane of the flow can thus be recovered by taking the envelop of the velocity data. We extract the flow direction, expressed by the angle $\alpha$, using average values at every channel position since no significant dependency of $\alpha$ on the $z$-direction was observed, nor expected. The resulting envelopes shown in Fig. 2(b) match the theoretical expectations, i.e., that the flow will develop from a flat velocity profile in the entrance region into a parabolic profile further downstream. Within the experimental error margin, $\alpha=\arctan \left(V_{y} / V_{x}\right) \approx 0$ everywhere except at the entrance of the channel and will therefore have little importance. In the following, we will thus focus the discussion on the velocity magnitude $V_{x y}=\sqrt{V_{x}^{2}+V_{y}^{2}}$. Here $V_{x}$ and $V_{y}$ are the velocity components in the $x$ - and $y$-directions, respectively. The example presented in Fig. 2 depicts a flow 
without any bubbles, and it is therefore stationary. Furthermore, typical flow velocities of $20 \mathrm{~ms}^{-1}$ ensure Reynolds numbers of the order of 400 and consequently a laminar flow regime. The presence of bubbles will add a periodic component in time. Postprocessing can be performed in the same way for each time step during the bubbling period, provided that the data set is sufficiently large.

\section{NUMERICS}

\section{A. Numerical scheme}

The incompressible Navier-Stokes equations are solved for both phases (liquid and gas) using a sharp-interface arbitrary Lagrangian-Eulerian finite element method [36,37]. To that end, the geometry of the channel is meshed with triangular Taylor-Hood elements [38]. For simplicity, however, the channel is assumed to be axisymmetric, i.e., the deviation from a circular cross section, as depicted in Fig. 1(b) or Fig. 2(c), is not taken into account. Implications of this assumption as well as of the assumption of incompressible liquids will be addressed in the discussion section. During the temporal integration, the mesh is always kept aligned with the moving liquid-gas interface, which requires the mesh to move together with the phase boundary. This is achieved by treating the mesh as a pseudoelastic body, which is deformed according to a displacement imposed via Lagrange multiplier fields at the moving interface [36,37]. The Lagrange multipliers ensure that the kinematic boundary condition is satisfied on both sides of the interface. When the mesh quality starts to suffer from the deformation, i.e., a single element either has doubled or halved its area with respect to the initial area, or the interior angles of the element drops below a threshold, the mesh is reconstructed and the pressure and velocity fields are subsequently interpolated to the new mesh. The implementation is performed using the open-source finite element library OOMPH-LIB [37]. The general model implementation has been successfully validated with simulations for a variety of other physical phenomena, ranging from evaporation [39], droplets bouncing in a stratified liquid due to buoyancy [40], and Marangoni flow and Leidenfrost droplets hovering on a bath [41].

While a benefit of the sharp-interface method is that the inclined walls at the outflow of the channel can be easily implemented, the topological changes that both phases undergo during the pinch-off events require a more sophisticated approach such as, e.g., in volume-of-fluid methods. Whenever the diameter of the gas domain falls below a critical threshold [here $2.5 \%$ of $w$ in Fig. 1(a)] at the imminent pinch-off point (local minimum in the gas domain diameter), the liquid-gas interface is dissected and both ends are reattached to the axis of symmetry, while ensuring volume conservation. The mesh is then rebuilt as described above.

The density in the simulation was set to $997 \mathrm{kgm}^{3}$ for the liquid and $1.25 \mathrm{kgm}^{3}$ for the gas. Dynamic viscosities were set to $1 \mathrm{mPas}$ and $0.0175 \mathrm{mPas}$ for the liquid and gas phase, respectively. Considering the microsecond timescale of bubble formation, we assume that the Tween 80 used in the experiments does not have sufficient time to significantly cover the interface during bubble pinch-off and the surface interfacial tension was therefore set to $72 \mathrm{mNm}^{-1}$. Indeed, no significant differences in bubbling frequency and size have been experimentally observed between bubble production with pure water and water-Tween solution. In the experiment, the surfactant thus mainly increases the stability of the bubbles against coalescence through its presence between the colliding bubbles in the expanding outlet channel, as previously shown for phospholipids [34].

A Poiseuille-like Dirichlet velocity profile was imposed at the liquid inlet and the volumetric inflow can be set to any desired flow rate. The gas is pushed into the channel via a normal traction boundary condition to impose the chosen gas overpressure. The outflow is an open, normal-stressfree, boundary.

\section{B. Validation of the numerical model}

The experimental channel geometry is described in Fig. 1(a) and corresponds to a hydraulic diameter of $18.4 \mu \mathrm{m}$. Consequently, for the numerical simulation with a circular cross section a diameter of $18.4 \mu \mathrm{m}$ was chosen. Furthermore, with this choice, the channel cross-sectional area 
(a)

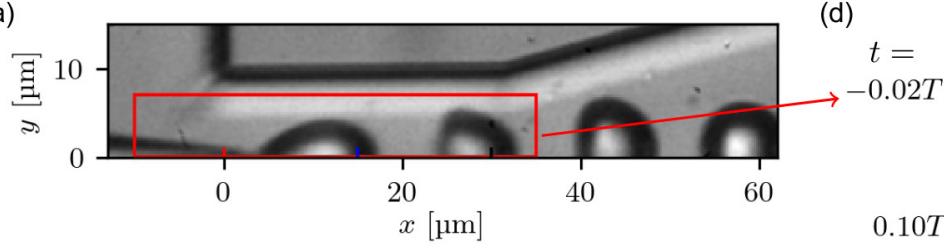

(b)

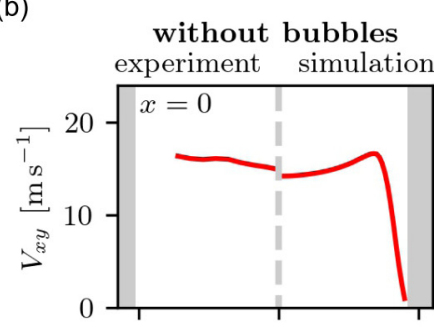

(c)

with bubbles
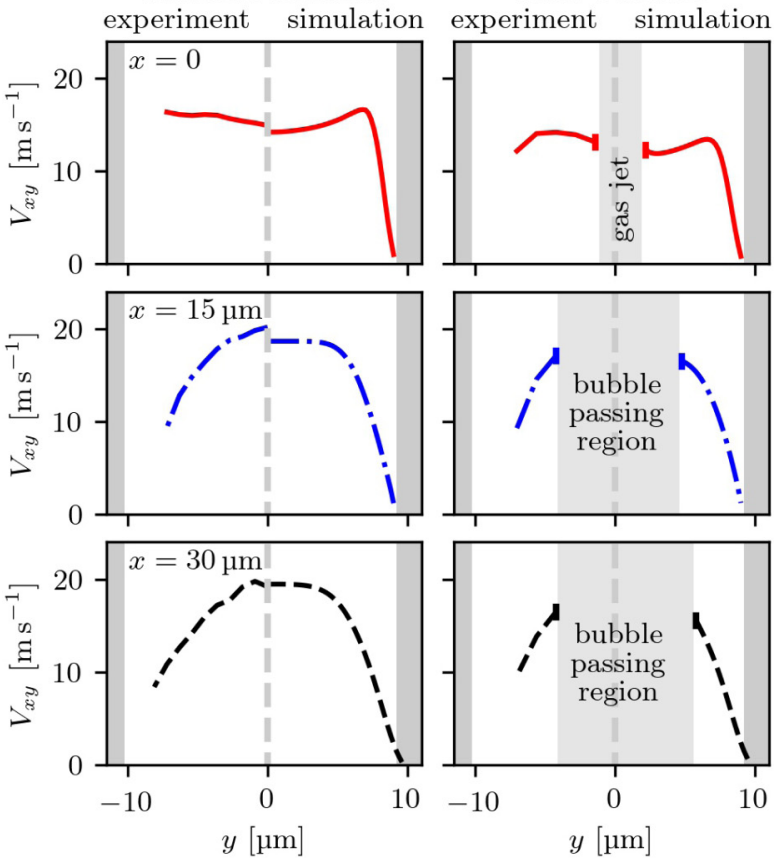

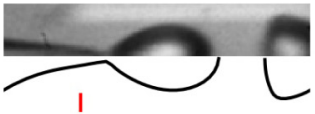

I

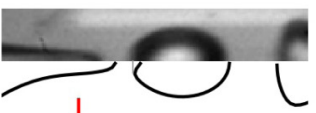

$0.22 T$

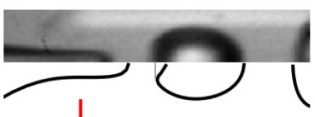

$0.34 T$

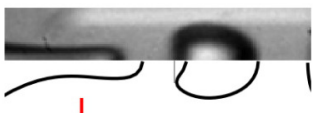

$0.46 T$

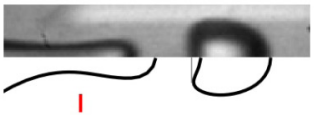

0.581

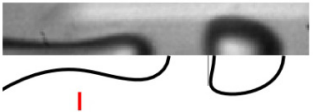

I

$0.70 T$

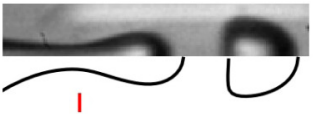

$0.82 T$

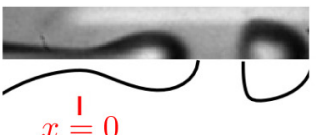

FIG. 3. Comparison between numerical model and experimental results. (a) Snapshot for reference. (b) Average flow profiles for experiments and simulations without bubbles. (c) Average flow profiles for experiments and simulations with bubbles. The center region where bubbles pass has been left on purpose in order not to induce any bias through averaging. (d) Temporal evolution of the jet tip and detached bubble in the flow-focusing channel, experiments (upper halves) compared to the numerically obtained gas-liquid interface (black line in the lower half plots). The thin gray lines that are added at the rear of the numerically obtained bubbles indicate the shape that would result from a projection of the bubble for comparison to the experimental shape.

is conserved within 3\% accuracy. Due to the differences in the precise channel geometry and due to the incompressibility of the simulation, some quantitative variations between experiments and simulations can be expected when considering a large range of parameters. However, as we will show in the following, simulations and experiments agree well for the parameter settings considered in this paper. We validate the numerical results using two specific cases.

Case (A): Liquid flow in the absence of bubbles at a flow rate of $0.2 \mathrm{mLmin}^{-1}$ is considered as the reference. Experimental and numerical curves are shown in Fig. 3(b). This case allows us to compare the experiments and the numerics to the analytical solutions for pipe flow. It is generally assumed that the flow develops from a flat profile in the inlet, to a parabolic profile corresponding to the wellknown Hagen-Poiseuille solution. In Fig. 3(b), the profile for $x \approx 0$ is not perfectly flat, but presents a maximum close to the channel wall, a phenomenon reported analytically, e.g., by Goldberg and Folk [42] and numerically by Dombrowski et al. [43]. The transition from a flat to a parabolic profile 
occurs over a typical distance defined as the entry length. For Reynolds numbers similar to the ones used here, $\operatorname{Re} \approx 400$, Dombrowski et al. [43] report an entry length of approximately 20 times the inlet diameter. Indeed, in the numerical case, it is visible that the parabolic shape is not fully reached, while the experimental results show a fully developed profile after a distance of merely 1.5 times the channel diameter. The difference between experiments and simulations may be due to slightly larger radial velocity components and irregularities in the channel geometry.

Case (B): The liquid flow is set to $158 \mu \mathrm{Lmin}^{-1}$ and the numerical gas pressure is set to $1.4 \mathrm{bar}$. This leads to the production of bubbles with a radius $R_{b}=5.5 \mu \mathrm{m}$ formed at a rate of $1.22 \times 10^{6}$ bubbles per second and traveling at approximately $20 \mathrm{~ms}^{-1}$. The gas flow rate can be calculated from the bubble size and bubbling frequency and gives $Q_{g}=40 \mu \mathrm{Lmin}^{-1}$. Adding the liquid flow rate yields a total of $Q_{t}=Q_{l}+Q_{g} \approx 200 \mu \mathrm{Lmin}^{-1}$. Thus, the total flow rates for cases (A) and (B) are comparable. This specific numerical setting provides a solution which is quantitatively comparable with the experiments when the chip is driven at a gas pressure of $2.7 \mathrm{bar}$. The difference in the set backing pressure can be explained by longer tubing and hence an increased total pressure drop in the experiments. Experimentally, $R_{b}$ ranges from 4.5 to $6.0 \mu \mathrm{m}$, where the bubble size inside the channel is oscillating due to compressibility effects and due to small variations between different recordings. Figure 3(c) shows the comparison between velocity profiles in the liquid phase, where the markers denote the edge of the bubbles. Figure 3(d) shows good agreement in bubble size, shape, and position between experiments and simulations for case (B). The largest difference concerns the size of the gas jet upstream of the channel entrance, i.e., for $x<0$. This difference may be explained by the fact that in the experimental case, the liquid streams through the two supply channels into the flow-focusing region, whereas the numerical case is axisymmetric resulting in an equally distributed inflow from all directions. The reference time $t=0$ corresponds to the moment of pinch-off.

\section{RESULTS}

\section{A. Average fields}

To understand the role of the flow and pressure components on the dynamics of the flow-focusing nozzle, it is convenient to separate their contributions in time-averaged fields and in oscillatory components. In this section we will first investigate the influence of bubbles on the average fields.

\section{Time-averaged velocity field}

A detailed look at the flow profiles in Fig. 3 reveals that the larger the bubbles, the steeper the velocity gradient near the channel wall. A first simple approach for the developed flow is to consider plug flow in the center of the channel at the location of the bubbles and to assume a solution of the Navier-Stokes equation for the region between the bubbles and the wall:

$$
V_{x}(r)=\left\{\begin{array}{l}
V_{b} \text { for } r \leqslant R^{*}, \\
-\frac{K r^{2}}{4}+A \ln \left(\frac{r}{w}\right)+B \text { for } R^{*} \leqslant r \leqslant w .
\end{array}\right.
$$

Here $w$ is the channel width, $r$ the radial coordinate and $R^{*}$ denotes the location of the transition between a parabolic and a flat profile. All variables of length $\left(r, w\right.$, and $\left.R^{*}\right)$ are in units of meter. The unknown velocity of the bubble $V_{b}$ as well as the constants $K, A$, and $B$ can be found using the boundary conditions

$$
\begin{aligned}
V_{x}(r & =w)=0, \\
V_{x}\left(r=R^{*}\right) & =V_{b}, \\
\frac{d}{d r} V_{x}\left(r=R^{*}\right) & =0,
\end{aligned}
$$



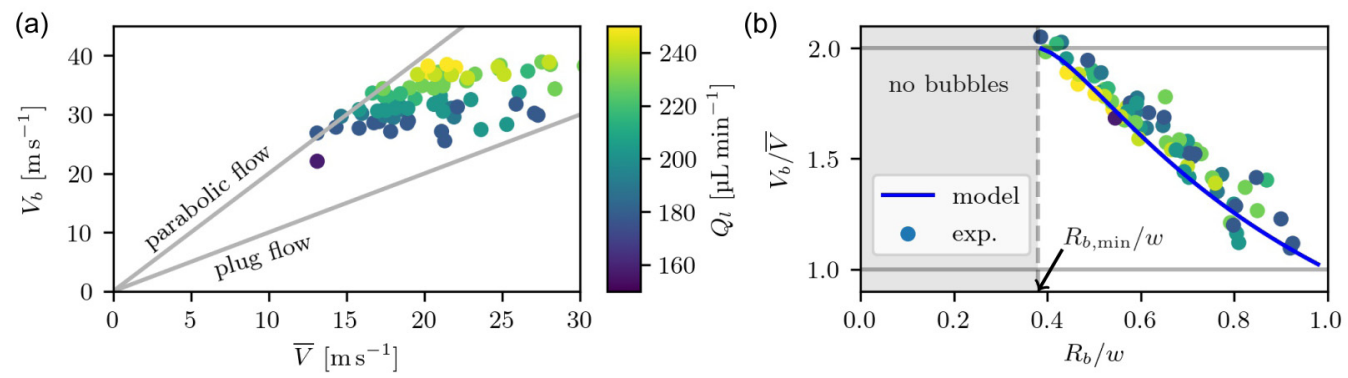

FIG. 4. (a) Experimental bubble velocity $V_{b}$ at the end of the flow-focusing channel as a function of the corresponding mean flow velocity $\bar{V}=\left(Q_{g}+Q_{l}\right) / A$ for a large number of experimental bubble sizes and liquid flow rates. The color code shows the different liquid flow rates used during bubble production. (b) Flow rate ratio $V_{b} / \bar{V}$ as a function of the bubble radius normalized by the channel radius and corresponding model from Eqs. (1) to (5). No bubbles smaller than $R_{b, \min }$ could be formed in the present flow-focusing device and within the parameter space explored.

and the integration

$$
Q_{t}=\int_{r=0}^{w} 2 \pi r V_{x}(r) d r
$$

where $Q_{t}=Q_{l}+Q_{g}$ is the known total (i.e., liquid plus gas) flow rate. Equation (2c) implies a free shear boundary condition and requires a negligible influence of surfactants on the liquid-gas boundary. Even though typical adsorption times for convective models are of the order of seconds $[44,45]$ and thus much larger than the present microsecond scale bubble production, typical times for a convective process as presented here are not commonly known. For this reason, the free shear boundary condition can also be explained by an expected light packing of surfactants at an early stage of bubble production in view of its final packing. In fact, it is known that a contrast agent bubble usually shrinks by a factor of approximately 2.5 [13] reducing the surface area to $15 \%$ of the initial size. Consequently the surface is $85 \%$ free upon formation and the molecules should be highly mobile, and thus we can assume that shear effects for such a lightly packed bubble are negligible [46]. The result from Eqs. (1) to (3) yields

$$
\begin{aligned}
K & =\frac{8 Q_{t}}{\pi\left(w^{2}-R^{* 2}\right)^{2}}, \\
A & =\frac{K}{2} R^{* 2}, \\
B & =\frac{K}{4} w^{2}, \\
V_{b} & =\frac{K}{4}\left[w^{2}-R^{* 2}+2 R^{* 2} \ln \left(\frac{w}{R^{*}}\right)\right] .
\end{aligned}
$$

Even though Eq. (4d) is not defined for the limiting cases, it tends towards a parabolic profile $V_{b}=2 \bar{V}$ for $R^{*} \rightarrow 0$ and a flat profile $V_{b}=\bar{V}$ for $R^{*} \rightarrow w$, where $\bar{V}=\left(Q_{t} / S\right)$ is the average velocity with the cross-sectional area $S=\pi w^{2}$. Both limiting cases are represented by the gray solid lines in Fig. 4(a), which shows the velocity of the bubbles versus the average flow velocity. The flow rate ratio as a function of the normalized bubble size is plotted in Fig. 4(b). It can be observed that, within the parameter space explored, the experimental data collapses on a single curve irrespective of the liquid flow rate applied. In addition, the blue line shows the theoretical 
considerations from eqs. 1 and $4 \mathrm{~d}$. Here the assumption of

$$
R^{*}=\left(R_{b}-R_{b, \min }\right)\left(\frac{1}{1-\frac{R_{b, \min }}{w}}\right)
$$

has been made, where $R_{b, \min } / w=0.38$ corresponds to the minimum bubble size observed experimentally; see Fig. 4(b). The expression of $R^{*}$ in Eq. (5) means a correction of the size of the plug flow area in the center of the channel. It takes into account that the plug flow region is smaller than the actual bubble size. This is in qualitative agreement with experimental and numerical observations, even though the time average in the bubble passing region [see Fig. 3(c)] has to interpreted with caution and is not shown in the present paper. Indeed, the time-dependent flow profile is much more complex, and Eqs. (1) to (5) are only a time-averaged approximation. An interesting feature of the model is that for minimum size bubbles, a parabolic average flow profile is assumed, which is in agreement with both the experimentally observed flow profiles such as presented in Fig. 3(b) and the bubble velocities in Fig. 4(b).

\section{Time-averaged pressure field}

The local pressure field is directly related to the flow velocity field via the Navier-Stokes equations and arises either from conversion of kinetic energy into pressure (volumetric potential energy) or from viscous losses. The derivatives of the velocity in the convection and diffusion terms of these equations, however, do not allow one to accurately recover the pressure field from the velocity measurements, owing to measurement noise. The pressure is therefore extracted from the numerical model. The corresponding pressure and velocity fields for case (A) without bubbles and case (B) with bubbles are shown in Figs. 5(a) and 5(b), respectively. The pressure profiles along the axial position $x$ are plotted in Fig. 5(c) for three radial positions $y=4,6$, and $8 \mu \mathrm{m}$.

In the inlet region of the flow-focusing channel, defined here by $-10 \mu \mathrm{m}<x<10 \mu \mathrm{m}$ and indicated as region I in Fig. 5(c) the liquid experiences a sharp pressure drop, which arises from both Bernoulli effects $\left(\Delta p_{\text {dyn }}\right)$ and viscous entry losses $\left(\Delta p_{\text {loss }}\right)$,

$$
\Delta p_{\text {I }}=\Delta p_{\text {dyn }}+\Delta p_{\text {loss }} \approx \frac{1}{2} \rho \bar{V}^{2}+K_{i} \frac{1}{2} \rho \bar{V}^{2},
$$

where $K_{i}$ the coefficient of resistance associated with the geometric shape of the inlet region, a number usually obtained empirically [47]. The velocity in the large channels upstream of the flowfocusing channel is much lower than in the narrow flow-focusing channel itself, and, therefore, it is neglected in the definition $\Delta p_{\text {dyn }}$. For case (A) without bubbles and with a liquid flow rate of $200 \mu \mathrm{Lmin}^{-1}, \Delta p_{\text {dyn }}=0.76$ bar, for case (B) with bubbles and a liquid flow rate of $158 \mu \mathrm{Lmin}^{-1}$, $\Delta p_{\text {dyn }}=0.47$ bar. In both cases this accounts for about half of the total pressure decrease [black points in Fig. 5(d)] in the inlet region I, the other half is attributed to viscous losses in the inlet region with $K_{i} \approx 1$. Strikingly, the pressure drop in region I, $\Delta p_{\mathrm{I}}$, does not significantly depend on the bubble size; see red points in Figs. 5(d) and 5(e). This suggests that, in the inlet region I, the gas jet has very little influence on the total flow behavior. The liquid flow rate is therefore the only relevant parameter to quantify the pressure drop in region I.

One can see from Fig. 5(c) that the pressure loss in region II is linear, which is consistent with the hydraulic pressure drop for a Poiseuille flow,

$$
\Delta p_{\text {linear }}=R_{H} q_{l},
$$

where $R_{H}=(8 \mu L) /\left(\pi w^{4}\right)$ is the hydraulic resistance [48]. The theoretical pressure drop along the $20 \mu \mathrm{m}$ channel length considered here is plotted by the black solid line in Fig. 5(f) for the case without bubbles. The theory slightly underestimates the numerically obtained pressure drop (black dots for simulations without bubbles). An explanation can be found in the slightly larger pressure gradient near the wall for the undeveloped flow in the simulation. In the case with bubbles, numerically obtained red dots in Fig. 5(f), the underestimation with respect to the theoretic 
(a)

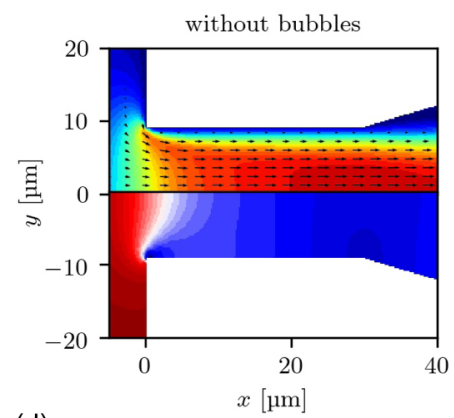

(d)

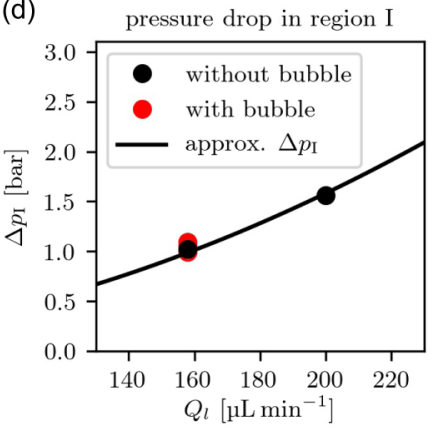

(b)

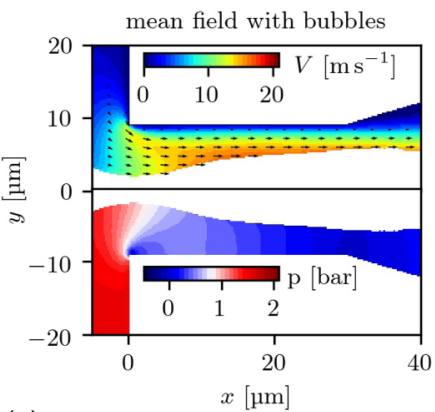

(e)
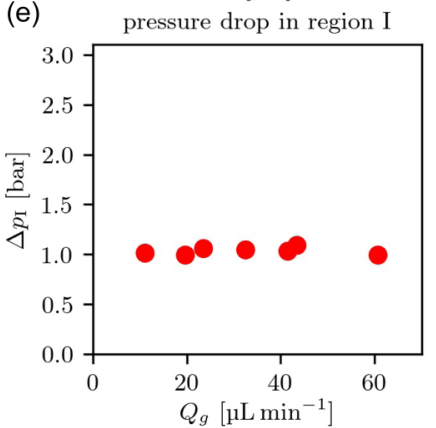

(c)

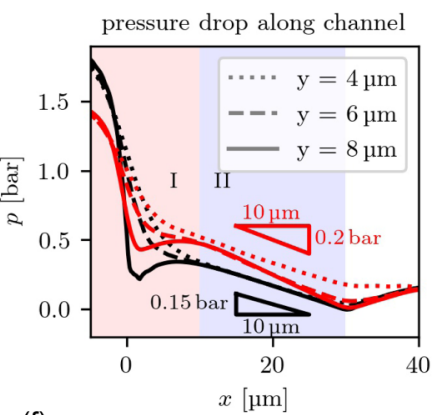

(f)

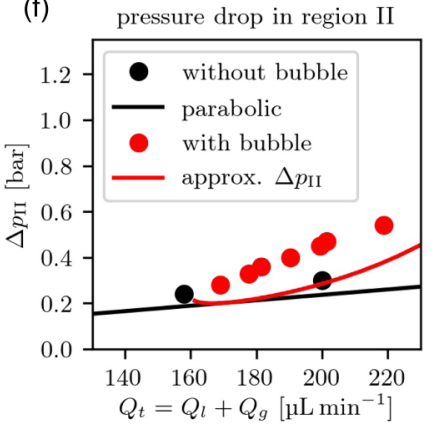

FIG. 5. (a, b) Average velocity (upper half) and pressure fields (lower half) for the case (A) without bubbles and the case (B) with bubbles, respectively. Note that for (b) the area where bubbles pass has intentionally been left blank in order not to induce any ambiguities in the definition of the mean velocity value. (c) Average pressure along three different lines parallel to the $x$-axis without bubbles (black line) and with bubbles (red line), both at a total flow rate $Q_{t}=Q_{l}+Q_{g}=200 \mu \mathrm{Lmin}^{-1}$. Note that for the radial position $y=4 \mu \mathrm{m}$ for the case with bubbles the time-averaged pressure includes moments where the gas phase is present, which leads to a slightly increased average pressure. As a reference, the minimum pressure appearing at about the channel end at $x \approx 30 \mu \mathrm{m}$ was set to zero. (d, e) Pressure drop in the inlet region I as a function of liquid flow rate and gas flow rate, respectively [approx. $\Delta p_{\mathrm{I}}$ : Eq. (6)]. (f) Pressure drop the region II as a function of total flow rate [parabolic: Eq. (7); approx. $\Delta p_{\mathrm{II}}$ : Eq. (8)].

estimation of a parabolic flow is even stronger. To take into account the deformation of the flow field as discussed in the above model, the pressure drop can be calculated to be

$$
\frac{d p}{d x}=\eta \frac{1}{r} \frac{\partial}{\partial r}\left(r \frac{\partial V_{x}}{\partial r}\right)=\frac{K}{\mu}=-\frac{8 \mu Q_{t}}{\pi\left(w^{2}-R^{* 2}\right)^{2}} .
$$

The result is shown in by the red line in Fig. 5(f). The remaining mismatch can mostly be attributed to a steeper velocity gradient close to the walls in the simulation for the undeveloped flow.

\section{B. Oscillatory fields}

Oscillatory fields are of particular interest here since they potentially result in cross-talk between parallel connected nozzles. It is therefore essential to quantify the oscillations and understand their origin.

\section{Oscillations in the gas phase}

Oscillations in the gas phase will have a direct impact on the liquid velocity inside the flowfocusing channel. The employed method of backlighting, where bubbles are imaged as black regions, makes it impossible, however, to use tracer particles to image the velocity of the gas jet. 


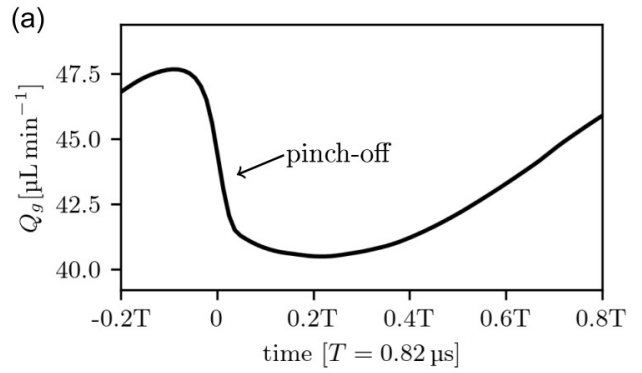

(b)

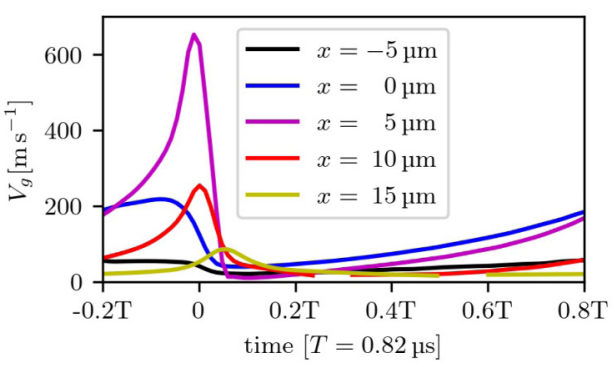

FIG. 6. Numerical oscillatory velocity in the gas phase for $y=0$ and different positions $x$ in the flowfocusing channel. (a) Gas flow rate as a function of time. (b) Gas velocity for different positions inside the gas thread (position of pinch-off is $x \approx 5 \mu \mathrm{m}$ ). Limitations of the incompressible simulations are discussed in the text.

We therefore have to rely on our numerical model. Figure 6(a) shows the gas flow rate, which is directly related to the gas velocity inside the gas supply channel. In this pressure-controlled system, the gas phase experiences velocity oscillations $[49,50]$ and consequently flow rate oscillations, here about $20 \%$ of the average value. To understand the origin of these gas flow oscillations, hydraulic losses, and Laplace pressure (see also Sec. IV B 3) need to be taken into account. After the moment of pinch-off, there is temporarily no jet neck, but a forward moving jet tip with a newly forming bubble. The Laplace pressure inside the bubble is decreasing in the beginning leading to an increased gas flow rate until hydraulic pressure losses become important and finally the new jet tip leads to a sudden increase of Laplace pressure, both decreasing the gas flow rate.

Around the position of pinch-off the thinning gas jet with a periodically variable diameter increases both the average gas velocity and the overlying oscillations [see Fig. 6(b)] leading to velocities of several hundred meters per second. While it is clear that such high velocities imply that our incompressible simulations do not completely represent the experimental case, supersonic flow velocities have been observed experimentally in other pinch-off studies [51], and we believe that our simulation provides sufficient qualitative insight into the predominant effects. Due to the compressibility of the gas before the jet neck, we can expect to observe lower flow velocities in the experimental case. Further aspects of the compressibility on the bubble dynamics will be addressed in the discussion section.

\section{Oscillatory velocity field}

Gas flow oscillations and the periodic generation of bubbles may lead to oscillations of the liquid velocity field as well. A simple way of exemplifying the unsteady nature of the liquid flow are the intersecting particle trajectories in Figs. 7(a) and 7(b), for experiments and numerical simulations, respectively.

The source of the phenomenon of intersecting particle trajectories becomes apparent when quantifying the flow field variations at the timescale of bubble generation. Velocity fluctuations in the continuous phase are plotted in Fig. 7(c) at four downstream locations in the channel and the radial position $y=6 \mu \mathrm{m}$, both for the experiments and for the simulations. For these positions, the velocity oscillates by 1 to $2 \mathrm{~ms}^{-1}$ for mean flow velocities of the order of $15 \mathrm{~ms}^{-1}$. Furthermore, Fig. 7(c) also shows that the velocity oscillations propagate along the channel axis, pushed along with the microbubble. A more extended look at the simulations in Fig. 7(f) reveals that in addition to the propagation along the channel axis, the oscillations are influenced by the bubble pinch-off at $t=0$ as well. Figure 7(f) shows the velocity (color) in the flow-focusing channel for a fixed radial position $y=6 \mu \mathrm{m}$ as a function of both the axial position $x$ and time $t$. The hatched areas indicate the presence of a bubble in the center of the channel. Figure 7(f) highlights that the region 
(a)

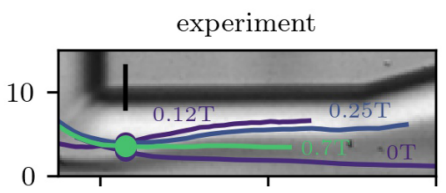

(b)

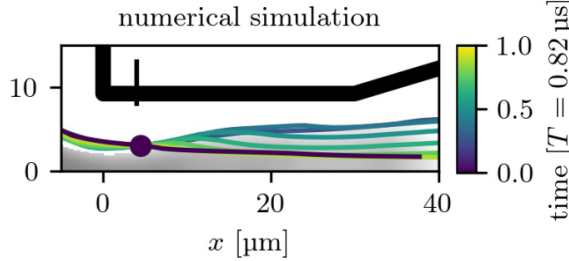

(d)

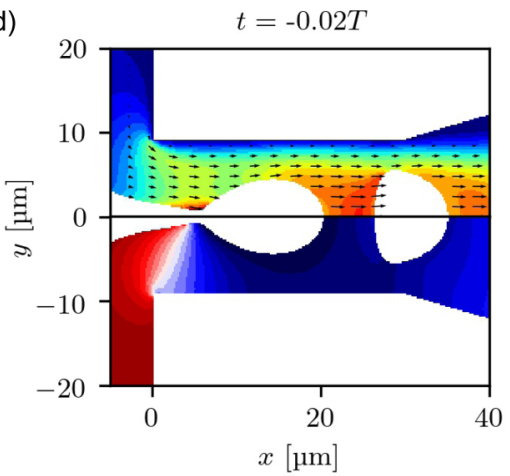

(f)

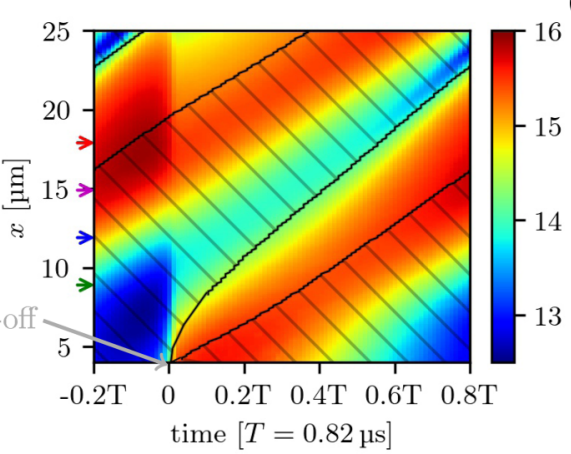

(c)

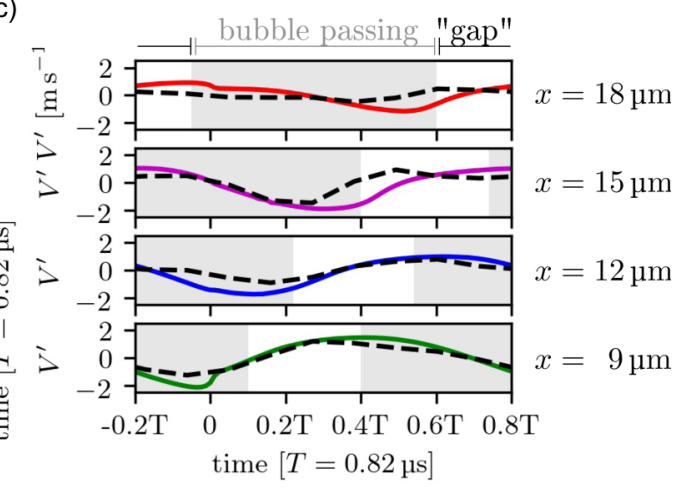

(e)

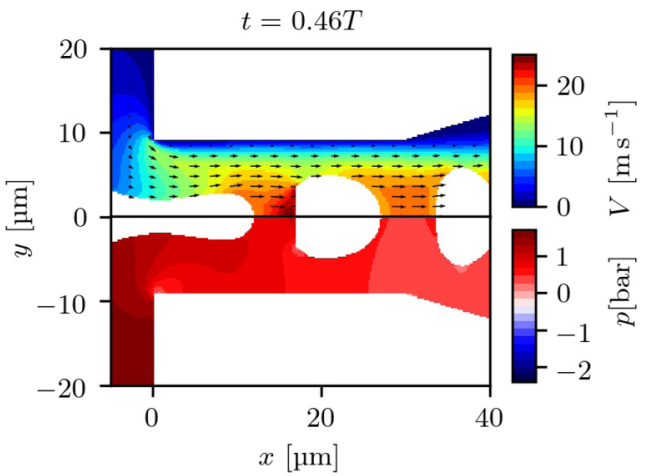

(g)

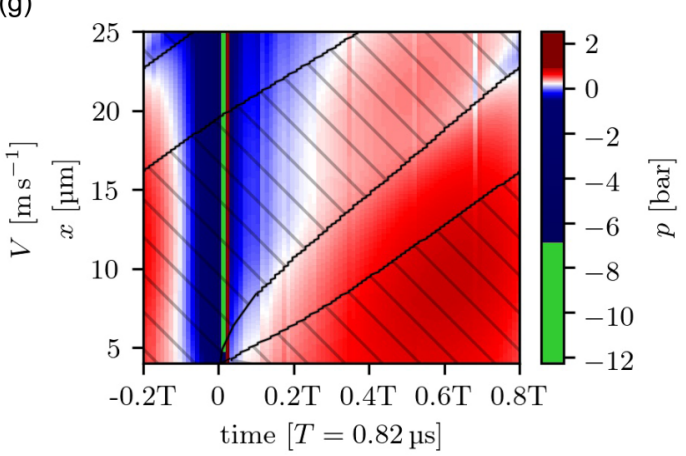

FIG. 7. Velocity and pressure oscillations in the flow-focusing channel. (a, b) Typical experimental and numerical particle trajectories highlight the time-dependent behavior at a given location. The time stamps correspond to the moment when the respective particles were passing the position indicated by the dots. (c) Velocity oscillations $V^{\prime}=V-\bar{V}$ for positions $y=5 \mu \mathrm{m}$ and $x$ given in the figure. The colored lines present the numerical results and the dashed black line the corresponding experimental data. (d, e) Velocity and pressure map for two instants during the bubbling period $T$. (f, g) Velocity $V$ and pressure $p$, respectively, for a fixed radial position $y=6 \mu \mathrm{m}$, different positions $x$, and different moments in time. The hatched areas correspond to the presence of a bubble in the respective channel sections $x$. The colored arrows in (f) indicate the positions in (c).

of increased velocity coincides with the front of the bubble. Furthermore, note that strong variations are present at the moment of bubble pinch-off, i.e., at $t=0$. 


\section{Oscillatory pressure field}

The neck shape is the origin of hydraulic losses and Laplace pressure. The pressure in the liquid can thus be seen as a consequence of the neck shape. The pressure inside the bubble is furthermore related to the pressure in the liquid via the Laplace pressure of the bubble. Orders of magnitude at the final stage before pinch-off (e.g., assuming a $0.1 \mu \mathrm{m}$ neck radius) are a 7 bar pressure difference between liquid and gas at the minimum neck radius due to Laplace pressure and a $0.35 \mathrm{bar} \mu \mathrm{m}^{-1}$ hydraulic pressure loss along the gas thread. In that sense the pressure impulse originates from the final, very short moment with a rapidly increasing Laplace pressure of the jet neck. After the moment of pinch-off, there is temporarily no jet neck, but a forward-moving jet tip with a newly forming bubble. Thus, it is the Laplace pressure of the bubble that is dominating; however, it decreases with increasing bubble size. This also explains the oscillations of the gas velocity: The Laplace pressure inside the bubble is decreasing in the beginning leading to an increased gas flow rate until hydraulic pressure losses become important where finally the new jet tip leads to a sudden decrease of the gas flow rate.

Oscillating gas and liquid velocities are bound to give rise to pressure oscillations. Figures $7(\mathrm{~d})$ and 7(e) show the pressure field at two specific moments of the bubble generation process, before and after bubble pinch-off (refer to Fig. 3 for the exact time stamps). The pressure varies by approximately 3 bar between these two moments. Figure $7(\mathrm{~g})$ displays the pressure for a fixed radial position $y=6 \mu \mathrm{m}$, different positions $x$ and different moments in time. One can in particular notice a strong pressure decrease around the moment of bubble pinch-off. This pressure drop is a consequence of the neck shape and Laplace pressure of the neck, which is increasing with decreasing jet radius (i.e., $0.1 \mu \mathrm{m}$ neck radius leading to 7 bar Laplace pressure). Through continuity and a relatively weak Laplace pressure of the bubble before pinch-off, the gas pressure inside the bubble also experiences a large pressure drop, which can be further explained by the hydraulic losses due to the very large gas velocities in the thinning jet. Between the bubble pinch-offs (here the time span $t=0.2 T$ to $0.8 T$ ), pressure values between 0 and 1 bar are observed in the channel. As for the average pressure, a general decrease along the $x$-axis is visible due to viscous losses already highlighted for the average field in Fig. 5. Furthermore, it can be observed that the region around the newly forming bubble corresponds to a region of higher pressure [bottom right area in Fig. 7(g)]. The use of an incompressible solver can be expected to have an influence on the exact shape and amplitude of the pressure pulse around $t=0$. If one accounts for compressibility, especially locally, the impulse will probably be less strong, and wider in time, thus effectively spreading the energy over a longer timescale. We believe, however, that the qualitative result of a strong pressure peak is representative for the real phenomenon. The phenomenon of acoustic emission at bubble pinch-off has been discussed in literature theoretically [52] and numerically [53] in order to understand the sound of rain, and later also for the pinch-off of bubble trains [54,55]. Among the different possible sources of sound proposed by Oğuz and Prosperetti [53], the most likely candidates for the large impulses at the moment of pinch-off observed in our simulations are the difference in Laplace pressure and the radial liquid inrush.

\section{DISCUSSION}

In the result section we present results on the average and oscillatory components of the velocities and pressures inside the flow-focusing device. While satisfactory agreement is obtained for the liquid velocities between the experimental and numerical method, it is important to keep in mind their respective limits. For the experimental results, we estimate the error on the absolute liquid velocities to be in the range $\pm 2 \ldots 3 \mathrm{~m} \mathrm{~s}^{-1}$. This results mainly (1) from small perturbations during the operating time of more than an hour, (2) from the particle tracking algorithm which occasionally outputs wrongly connected particle positions and therefore incorrect velocities which increase the number of outliers, and (3) from the need to extract velocities via an envelope due to detection of 
particles in the entire channel. Nonetheless, relative velocity oscillations can be detected with higher accuracy.

Numerically, the main limitation arises through the use of an incompressible solver. As already discussed in Sec. IV B 1, this leads in particular to an overestimation of the gas flow velocities before the position of pinch-off. The simulated incompressibility of the gas also leads to a constant bubble volume once the bubble is pinched off, while in the experimental case the bubble radius is oscillating by about $10 \%$ while traveling down the flow-focusing channel. This type of dynamics is known from the Rayleigh-Plesset-type description for a free bubble [56]. In the present study, however, it is further influenced by the specific liquid-gas interaction confined in the channel. Another important limitation of the incompressible solver for both the liquid and gas phase is an infinite speed of sound and consequently the lack to correctly represent acoustic effects. In the real scenario, low compressibility effects give rise to the wave equation, and thus to the propagation of an acoustic impulse. Due to the small scale of the chip, the time to propagate through the $20 \mu \mathrm{m}$ long channel would still be very short $\sim 15 \mathrm{~ns}$. The time to travel between several parallelized channels would be of the order of a few hundred ns.

Lastly, it should be noted that both the completely circular numerical cross section and the flattened experimental cross section differ from other typical channel types, in particular those with rectangular cross sections. Calculating the pressure drop in rectangular channels is more complex (see [48] for more details) and taking into account bubbles is expected to be less straightforward than for a circular cross section. Especially if the bubble nearly fills the complete channel, the effect of flow in the sharp corners becomes important [57]. In general, however, the influence of the corner flow gets weaker towards the center of the channel, where our small bubbles are passing $[48,58]$. We thus believe, that our results will also apply to other channel geometries, provided that the channel aspect ratio remains of the order of one and that bubbles remain small compared to the channel size.

Our results highlight the oscillatory nature of the velocity and pressure inside a flow-focusing device, where we observe oscillations in the gas flow rate by numerical simulation, in agreement with earlier work $[49,50]$. Even though compressibility effects may lead to lower maximum velocities, values of the order of the speed of sound have been observed as shown before experimentally [51]. The collapsing jet neck leads to a pressure impulse as confirmed by numerical simulations. Once again, the exact pressure amplitude may differ from the compressible case, but our findings are in agreement with the observation of acoustic emission at the moment of bubble pinch-off found in other systems [52-55]. The oscillating gas phase leads to oscillations of the liquid velocity. To the best of our knowledge these oscillations have not been studied before.

Our study gives detailed insight into the physical processes linked to the bubble production inside a flow-focusing chip. It highlights how both, the average and the oscillatory field are influenced by the presence of the bubbles. These results can be used in further studies to understand potential communication between parallelized channels that could lead to a decreased monodispersity [19,21]. The insights into the dynamic flow fields investigated in this paper will therefore be instrumental to a successful design as to drive parallelized high-production rate nozzles for microfluidic industrial applications.

\section{CONCLUSIONS}

The present study shows the time-averaged and time-resolved velocity field and pressure field in a flow-focusing device. Particle tracking has been used to evaluate the velocity field experimentally, and numerical simulations were used to provide insight in both the velocity and pressure fields. We can observe three types of oscillations. (1) As we have a pressure-driven gas feeding, the gas velocity is oscillating as a result of the changing Laplace pressure at the tip of the jet upon bubble pinch-off. This leads to velocity maxima of the order of several hundred meters per second in the jet neck region. (2) Oscillations can also be observed for the liquid velocity. They are closely linked to the presence of the bubbles, propagate with the velocity of the bubble and amount to up to $25 \%$ of the total velocity in the case studied here in detail. (3) Pressure oscillations in the liquid 
phase are dominated by a strong negative pressure impulse linked to the periodic bubble pinch-off. Understanding these different types of oscillations will be a valuable asset to understand the role of cross-talk for parallelized flow-focusing nozzles. Experiments and/or simulations of such coupled systems will, however, be necessary to fully understand the importance of the respective oscillatory components in a coupled device. In addition to the results on the oscillatory flow, the present study provides insight into the effect of bubbles on the time-averaged liquid flow and pressure field inside a flow-focusing chip. The velocity profile can be approximated by a simple analytic expression, while the presence of bubbles leads to a significant viscous pressure drop in the flow-focusing channel.

\section{ACKNOWLEDGMENTS}

This project is partly financed by Holland High Tech with a public-private partnership allowance in the top sector High Tech Systems and Materials (HTSM) and by Bracco Suisse S.A. G.L. acknowledges funding from the 4TU Precision Medicine program supported by High Tech for a Sustainable Future, a framework commissioned by the four Universities of Technology of the Netherlands. T.S. acknowledges funding from the Max Planck Center for Complex Fluid Dynamics.

[1] E. C. Unger, T. Porter, W. Culp, R. Labell, T. Matsunaga, and R. Zutshi, Therapeutic applications of lipid-coated microbubbles, Adv. Drug Delivery Rev. 56, 1291 (2004).

[2] I. Lentacker, I. De Cock, R. Deckers, S. De Smedt, and C. Moonen, Understanding ultrasound induced sonoporation: Definitions and underlying mechanisms, Adv. Drug Delivery Rev. 72, 49 (2014).

[3] K. Hynynen, N. McDannold, N. Vykhodtseva, and F. A. Jolesz, Noninvasive MR imaging-guided focal opening of the blood-brain barrier in rabbits, Radiology 220, 640 (2001).

[4] T. R. Porter and F. Xie, Ultrasound, microbubbles, and thrombolysis, Prog. Cardiovasc. Dis. 44, 101 (2001).

[5] M. Versluis, E. Stride, G. Lajoinie, B. Dollet, and T. Segers, Ultrasound contrast agent modeling: A review, Ultrasound Med. Biol. 46, 2117 (2020).

[6] P. Frinking, T. Segers, Y. Luan, and F. Tranquart, Three decades of ultrasound contrast agents: A review of the past, present and future improvements, Utrasound Med. Biol. 46, 892 (2020).

[7] E. Talu, K. Hettiarachchi, S. Zhao, R. L. Powell, A. P. Lee, M. L. Longo, and P. A. Dayton, Tailoring the size distribution of ultrasound contrast agents: Possible method for improving sensitivity in molecular imaging, Mol. Imag. 6, 7290 (2007).

[8] T. Segers, P. Kruizinga, M. P. Kok, G. Lajoinie, N. De Jong, and M. Versluis, Monodisperse versus polydisperse ultrasound contrast agents: Non-linear response, sensitivity, and deep tissue imaging potential, Ultrasound Med. Biol. 44, 1482 (2018).

[9] A. Helbert, E. Gaud, T. Segers, C. Botteron, P. Frinking, and V. Jeannot, Monodisperse versus polydisperse ultrasound contrast agents: In vivo sensitivity and safety in rat and pig, Ultrasound Med. Biol. 46, 3339 (2020).

[10] T. Segers and M. Versluis, Acoustic bubble sorting for ultrasound contrast agent enrichment, Lab Chip 14, 1705 (2014).

[11] J. A. Feshitan, C. C. Chen, J. J. Kwan, and M. A. Borden, Microbubble size isolation by differential centrifugation, J. Colloid. Interface Sci. 329, 316 (2009).

[12] J. Rodríguez-Rodríguez, A. Sevilla, C. Martínez-Bazán, and J. M. Gordillo, Generation of microbubbles with applications to industry and medicine, Annu. Rev. Fluid Mech. 47, 405 (2015).

[13] T. Segers, L. De Rond, N. de Jong, M. Borden, and M. Versluis, Stability of monodisperse phospholipidcoated microbubbles formed by flow-focusing at high production rates, Langmuir 32, 3937 (2016).

[14] B. van Elburg, G. Collado-Lara, G.-W. Bruggert, T. Segers, M. Versluis, and G. Lajoinie, Feedbackcontrolled microbubble generator producing $1 \times 106$ monodisperse bubbles per second, Rev. Sci. Instrum. 92, 035110 (2021). 
[15] M. T. Sullivan and H. A. Stone, The role of feedback in microfluidic flow-focusing devices, Philos. Trans. R. Soc. A: Math. Phys. Eng. Sci. 366, 2131 (2008).

[16] D. Conchouso, D. Castro, S. Khan, and I. Foulds, Three-dimensional parallelization of microfluidic droplet generators for a litre per hour volume production of single emulsions, Lab Chip 14, 3011 (2014).

[17] H.-H. Jeong, V. R. Yelleswarapu, S. Yadavali, D. Issadore, and D. Lee, Kilo-scale droplet generation in three-dimensional monolithic elastomer device (3D MED), Lab Chip 15, 4387 (2015).

[18] M. Hashimoto, S. S. Shevkoplyas, B. Zasońska, T. Szymborski, P. Garstecki, and G. M. Whitesides, Formation of bubbles and droplets in parallel, coupled flow-focusing geometries, Small 4, 1795 (2008).

[19] C. Jiang, X. Li, Q. Jin, T. Chen, Z. Wang, and H. Zheng, Mass production of monodisperse ultrasound contrast microbubbles in integrated microfluidic devices, in Proceedings of the 2010 4th International Conference on Bioinformatics and Biomedical Engineering, Chengdu, China (IEEE, Piscataway, NJ, 2010), pp. 1-4.

[20] J. L. Chen, A. H. Dhanaliwala, S. Wang, and J. A. Hossack, Parallel output, liquid flooded flow-focusing microfluidic device for generating monodisperse microbubbles within a catheter, in Proceedings of the 2011 IEEE International Ultrasonics Symposium, Orlando, FL (IEEE, Piscataway, NJ, 2011), pp. 160163.

[21] M. R. Kendall, D. Bardin, R. Shih, P. A. Dayton, and A. P. Lee, Scaled-up production of monodisperse, dual layer microbubbles using multi-array microfluidic module for medical imaging and drug delivery, Bubble Sci. Eng. Technol. 4, 12 (2012).

[22] H.-H. Jeong, S. Yadavali, D. Issadore, and D. Lee, Liter-scale production of uniform gas bubbles via parallelization of flow-focusing generators, Lab Chip 17, 2667 (2017).

[23] H.-H. Jeong, Z. Chen, S. Yadavali, J. Xu, D. Issadore, and D. Lee, Large-scale production of compound bubbles using parallelized microfluidics for efficient extraction of metal ions, Lab Chip 19, 665 (2019).

[24] M. Hashimoto, P. Garstecki, and G. M. Whitesides, Synthesis of composite emulsions and complex foams with the use of microfluidic flow-focusing devices, Small 3, 1792 (2007).

[25] W. Li, E. W. Young, M. Seo, Z. Nie, P. Garstecki, C. A. Simmons, and E. Kumacheva, Simultaneous generation of droplets with different dimensions in parallel integrated microfluidic droplet generators, Soft Matter 4, 258 (2008).

[26] M. Shirota, T. Sanada, A. Sato, and M. Watanabe, Formation of a submillimeter bubble from an orifice using pulsed acoustic pressure waves in gas phase, Phys. Fluids 20, 043301 (2008).

[27] M. Shirota, T. Imamura, and M. Kameda, Formation of single bubbles from a submerged orifice using pulsed ultrasound waves, J. Fluid Sci. Tech. 3, 183 (2008).

[28] K. Mu, T. Si, E. Li, R. X. Xu, and H. Ding, Numerical study on droplet generation in axisymmetric flow focusing upon actuation, Phys. Fluids 30, 012111 (2018).

[29] C. Yang, R. Qiao, K. Mu, Z. Zhu, R. X. Xu, and T. Si, Manipulation of jet breakup length and droplet size in axisymmetric flow focusing upon actuation, Phys. Fluids 31, 091702 (2019).

[30] B. R. Mutlu, J. F. Edd, and M. Toner, Oscillatory inertial focusing in infinite microchannels, Proc. Natl. Acad. Sci. USA 115, 7682 (2018).

[31] G. Vishwanathan and G. Juarez, Inertial focusing in planar pulsatile flows, J. Fluid Mech. 921, R1 (2021).

[32] M. Köhler, Isotropic etching, in Encyclopedia of Microfluidics and Nanofluidics, edited by Li. Dongqing (Springer Science \& Business Media, Springer, Boston, MA, 2008), pp. 877-884.

[33] T. Segers, E. Gaud, M. Versluis, and P. Frinking, High-precision acoustic measurements of the nonlinear dilatational elasticity of phospholipid coated monodisperse microbubbles, Soft Matter 14, 9550 (2018).

[34] T. Segers, D. Lohse, M. Versluis, and P. Frinking, Universal equations for the coalescence probability and long-term size stability of phospholipid-coated monodisperse microbubbles formed by flow focusing, Langmuir 33, 10329 (2017).

[35] J. C. Crocker and D. G. Grier, Methods of digital video microscopy for colloidal studies, J. Colloid. Interface Sci. 179, 298 (1996).

[36] R. A. Cairncross, P. R. Schunk, T. A. Baer, R. R. Rao, and P. A. Sackinger, A finite element method for free surface flows of incompressible fluids in three dimensions. Part I. Boundary fitted mesh motion, Int. J. Numer. Methods Fluids 33, 375 (2000). 
[37] M. Heil and A. L. Hazel, oomph-lib-An Object-Oriented Multi-Physics finite-element Library, Lect. Notes Comput. Sci. Eng. 53, 19 (2006).

[38] C. Taylor and P. Hood, A numerical solution of the Navier-Stokes equations using the finite element technique, Comput. Fluids 1, 73 (1973).

[39] Y. Li, C. Diddens, T. Segers, H. Wijshoff, M. Versluis, and D. Lohse, Evaporating droplets on oil-wetted surfaces: Suppression of the coffee-stain effect, Proc. Natl. Acad. Sci. USA 117, 16756 (2020).

[40] Y. Li, C. Diddens, A. Prosperetti, K. L. Chong, X. Zhang, and D. Lohse, Bouncing Oil Droplet in a Stratified Liquid and Its Sudden Death, Phys. Rev. Lett. 122, 154502 (2019).

[41] A. Gauthier, C. Diddens, R. Proville, D. Lohse, and D. van der Meer, Self-propulsion of inverse Leidenfrost drops on a cryogenic bath, Proc. Natl. Acad. Sci. USA 116, 1174 (2019).

[42] I. S. Goldberg and R. T. Folk, Solutions for steady and nonsteady entrance flow in a semi-infinite circular tube at very low Reynolds numbers, SIAM J. Appl. Math. 48, 770 (1988).

[43] N. Dombrowski, E. Foumeny, S. Ookawara, and A. Riza, The influence of Reynolds number on the entry length and pressure drop for laminar pipe flow, Can. J. Chem. Eng. 71, 472 (1993).

[44] A. Bąk and W. Podgórska, Interfacial and surface tensions of toluene/water and air/water systems with nonionic surfactants Tween 20 and Tween 80, Colloids Surf., A 504, 414 (2016).

[45] C.-H. Chang and E. I. Franses, Adsorption dynamics of surfactants at the air/water interface: A critical review of mathematical models, data, and mechanisms, Colloids Surf., A 100, 1 (1995).

[46] P. Marmottant, S. Van Der Meer, M. Emmer, M. Versluis, N. De Jong, S. Hilgenfeldt, and D. Lohse, A model for large amplitude oscillations of coated bubbles accounting for buckling and rupture, J. Acoust. Soc. Am. 118, 3499 (2005).

[47] M. E. Steinke and S. G. Kandlikar, Single-phase liquid friction factors in microchannels, Int. J. Therm. Sci. 45, 1073 (2006).

[48] H. Bruus, Theoretical Microfluidics (Oxford University Press, Oxford, 2007).

[49] P. M. Wilkinson and L. L. Van Dierendonck, A theoretical model for the influence of gas properties and pressure on single-bubble formation at an orifice, Chem. Eng. Sci. 49, 1429 (1994).

[50] J. M. Gordillo, A. Sevilla, and C. Martínez-Bazán, Bubbling in a co-flow at high Reynolds numbers, Phys. Fluids 19, 077102 (2007).

[51] S. Gekle, I. R. Peters, J. M. Gordillo, D. van der Meer, and D. Lohse, Supersonic Air Flow Due to Solid-Liquid Impact, Phys. Rev. Lett. 104, 024501 (2010).

[52] M. S. Longuet-Higgins, An analytic model of sound production by raindrops, J. Fluid Mech. 214, 395 (1990).

[53] H. N. Oğuz and A. Prosperetti, Numerical calculation of the underwater noise of rain, J. Fluid Mech. 228, 417 (1991).

[54] R. Manasseh, R. LaFontaine, J. Davy, I. Shepherd, and Y.-G. Zhu, Passive acoustic bubble sizing in sparged systems, Exp. Fluids 30, 672 (2001).

[55] J. Liu, W. Wang, N. Chu, D. Wu, and W. Xu, Numerical simulations and experimental validation on passive acoustic emissions during bubble formation, Appl. Acoust. 130, 34 (2018).

[56] W. Lauterborn, Numerical investigation of nonlinear oscillations of gas bubbles in liquids, J. Acoust. Soc. Am. 59, 283 (1976).

[57] V. van Steijn, C. R. Kleijn, and M. T. Kreutzer, Flows Around Confined Bubbles and Their Importance in Triggering Pinch-Off, Phys. Rev. Lett. 103, 214501 (2009).

[58] M. K. Moharana and S. Khandekar, Generalized formulation for estimating pressure drop in fullydeveloped laminar flow in singly and doubly connected channels of non-circular cross-sections, Comput. Methods Appl. Mech. Eng. 259, 64 (2013). 